\title{
A method for examining the operant self-administration of respirable pharmaceuticals in rodents
}

\author{
JOHN M. ROLL, BRIAN P. KELLY, FRANCIS BENJAMIN, and RONALD E. SEE \\ Washington State University, Pullman, Washington
}

\begin{abstract}
Many different drugs of abuse are administered via an inhalation route. However, no pragmatic, infraprimate model has been developed to assess the self-administration of these pharmaceutical agents in the laboratory setting. This paper describes an apparatus designed to assess the selfadministration of these pharmaceutical agents in a rodent model.
\end{abstract}

Several of the most frequently abused drugs are often administered via an inhalation route (i.e., nicotine, tetrahydrocannabinol, opium, cocaine, and methamphetamine). Chaiken (1993) has described the use of these inhalable drugs as being at "epidemic" levels in the United States. Unfortunately, basic and applied research in this area have been driven by the "epidemic" itself instead of having predicted the "epidemic." One reason that research in this area may have been slowed is the lack of a pragmatic laboratory model to study self-administrative behavior of these abused, respirable pharmaceuticals.

Primate species are an ideal choice for an animal model with which to assess the self-administration of these agents. Yet although continued use of the primate model is indispensable, current restrictions on primate use, combined with the high cost of maintaining primates in a laboratory setting, renders the primate model unpragmatic for certain applications and for researchers in smaller facilities.

The apparatus described here may help alleviate some of the aforementioned problems. It is relatively cheap and simple to construct, and it is intended for the use of laboratory rodents as subjects. In addition to being practical, the apparatus facilitates the comparison of self-administration data and data for forced administration of respirable pharmaceuticals. A growing literature concerning the forced administration of these drugs is appearing (e.g., Boni, Barr, \& Martin, 1991; Burchfield, Abrams, Miller, \& DeVane, 1991), and it will be important to compare the results from those studies with the results of studies on the self-administration of the same drugs.

The authors wish to thank Kelly S. Johnson for assistance with this project. We also wish to acknowledge N. John Castellan, Jr.'s thoughtful comments on an earlier version of this paper. Cocaine for the data presented in figures 3 and 4 was generously provided to R.E.S. by the National Institute of Drug Abuse. Correspondence should be addressed to J. M. Roll, Department of Psychology, Washington State University, Pullman, WA 99164-4820.
Two methods are currently available that approximate an adequate self-administration model; both of them, however, fall somewhat short. Prusaczyk and Fischer (1983) developed a small-animal inhalation chamber for administering respirable drugs. With their chamber, it is possible to make delivery of the drug contingent on the animal's entering a certain portion of the chamber. However, the operant, in a procedure of this type, is confounded with a classically conditioned place preference, and it is difficult to claim that the animal is actually selfadministering.

Another, more successful, procedure was developed by Jaffe, Sharpe, and Jaffe (1989). These researchers were able to shape rats to leverpress for nebulized sufentanil, an opioid agonist, in a specially designed chamber. But a potential problem with external validity occurs when one uses Jaffe et al.'s method. Most drugs of abuse which are inhaled are pyrolized, not nebulized. This makes comparison across delivery methods necessary. The method to be described here can accommodate either a nebulization or a pyrolization procedure, facilitating a wider variety of research activities.

\section{CONSTRUCTION}

Figures 1 and 2 detail the appropriate construction of the self-administration apparatus. The apparatus is designed to facilitate in vivo microdialysis while the rodent is self-administering. Construction of the apparatus is relatively straightforward. Replication of the design indicated by the schematics in Figures 1 and 2 will result in a functional apparatus.

The specifications in Figures 1 and 2 are for use of the apparatus with rats weighing between 250 and $500 \mathrm{~g}$. For rodents of different sizes, it will be necessary to change the size of certain portions of the apparatus. Of particular importance will be the length and width of the drugadministration chamber (DAC). These measurements should be tailored to the size of the rodent utilized. 


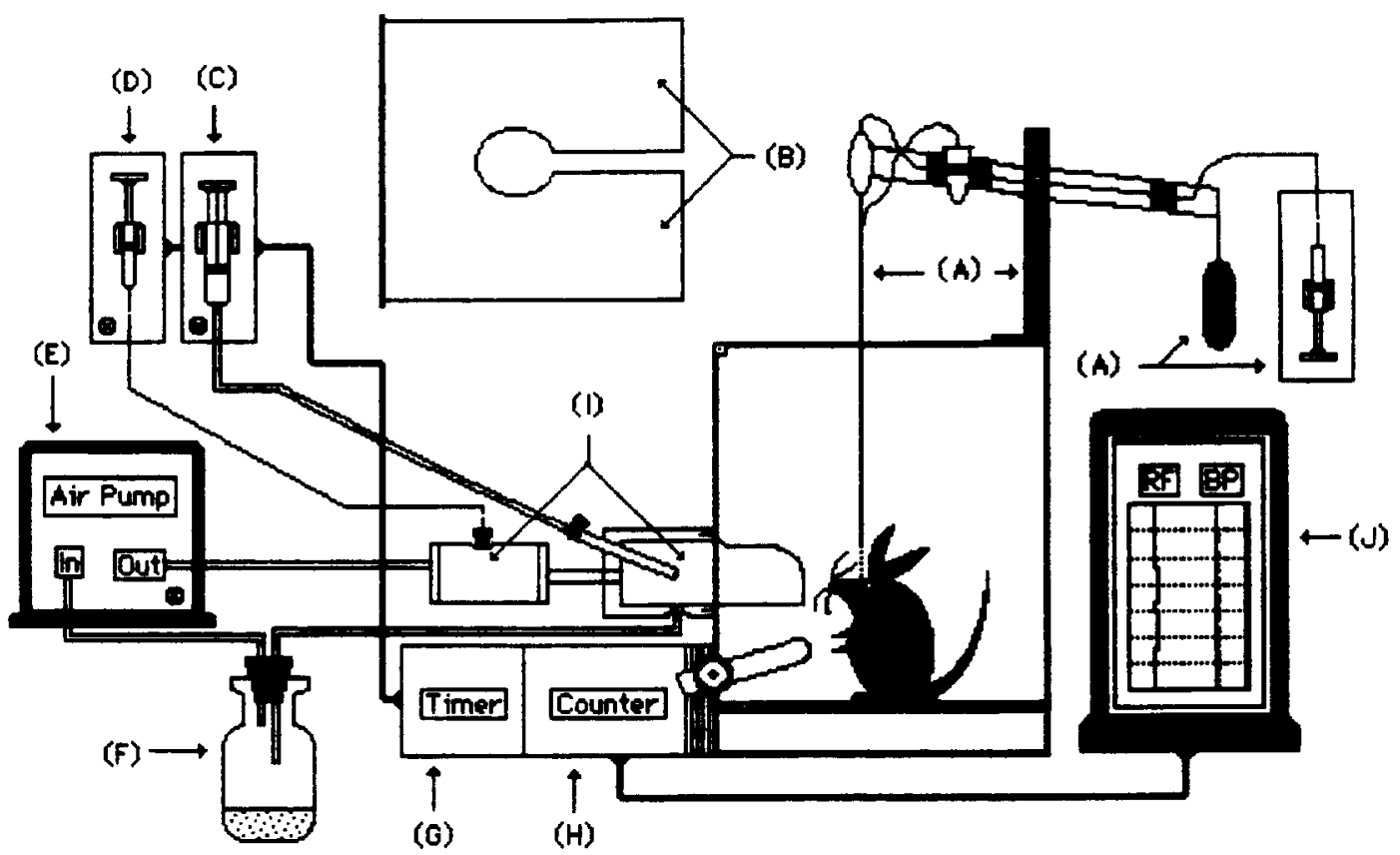
(A) Microdialysis Equipment
(B) Top of Operant Box
(C) Syringe Pump (Reinforcer) ( $35 \mathrm{ml}$ Syringe)
(D) Syringe Pump (Drug) (3-5 ml Syringe)
(E) Air Pump

(F) Unused Reinforcer Resevior

(G) Timer

(H) Counter
(1) Drug Administration Appar atus (See Close-Up)

(J) Chart Recorder

Figure 1. Schematic representation of respiratory drug administration apparatus. Apparatus is depicted for conducting concurrent microdialysis.

The air pump ( $E$ in Figure 1) can be of any type that delivers a constant stream of air when activated. It is more difficult to shape rats to respond when the pump delivers intermittent "puffs" of air. With the use of a nebulizer/air pump combination (DeVILBISS Model 5610D) it is possible to disconnect the drug administration apparatus (I in Figure 1) and use the remainder of the device to examine self-administration of nebulized drugs.

The apparatus, as depicted in Figure 1, is equipped with a cumulative type chart recorder. This could be replaced with a tape counter. It could also be replaced or augmented by attaching it to a computer with any of the readily available operant chamber interfaces on the market. This would facilitate the recording of more complicated molecular behavioral measures.

The counter and timer ( $\mathrm{G}$ and $\mathrm{H}$ in Figure 1) are attached to the two syringe pumps; one syringe pump delivers liquid reinforcer, while the other delivers drug in solution. The counter/timer setup can be any of a number of commercially available units (e.g., Lafayette No. 55040). With the counter/timer apparatus, it is possible to program a wide variety of schedule parameters.

\section{SHAPING PROTOCOL}

A fairly typical shaping by successive approximations procedure is used to shape self-administrative behavior in the apparatus. There are, however, several procedures that promote rapid shaping. It is advisable to shape the leverpressing behavior with food or water reinforcement before shifting to drug reinforcement. This will save on the amount of drug used. During this initial shaping phase, unconsumed reinforcer should not be allowed to pool in the drug administration apparatus. This is prevented by using the vacuum apparatus ( $E$ and $F$ in Figure 1) to remove uncollected reinforcer.

Once the leverpressing behavior has stabilized for food or water reinforcement, the drug volatilization chamber should be turned on. This will warm the air that is blown into the drug administration chamber. A transient decrease in responding will most likely be observed. After responding has restabilized, the vehicle in which the drug is to be dissolved should be introduced into the drug volatilization chamber. Again, a transient decrease in responding will be observed. 


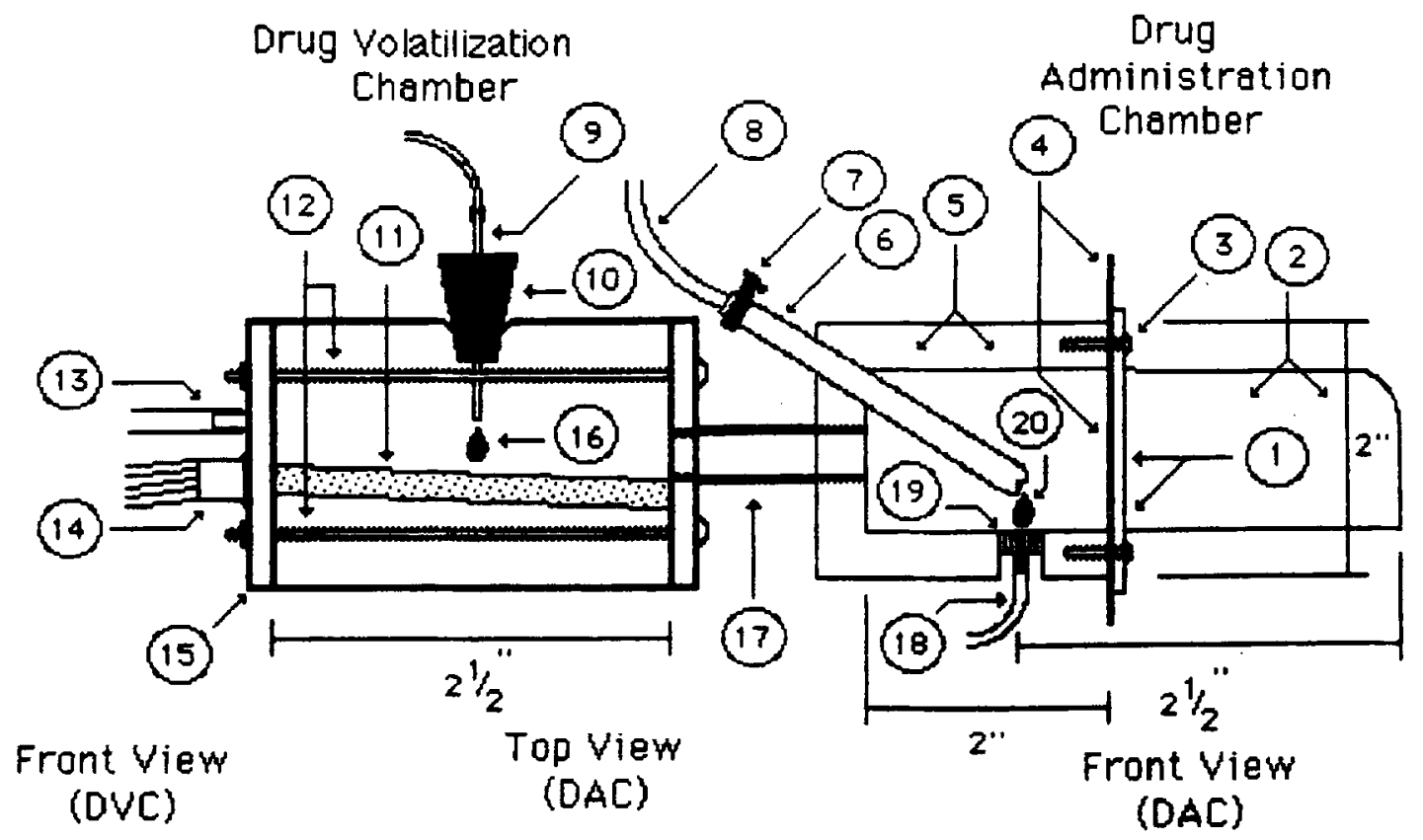

(12)
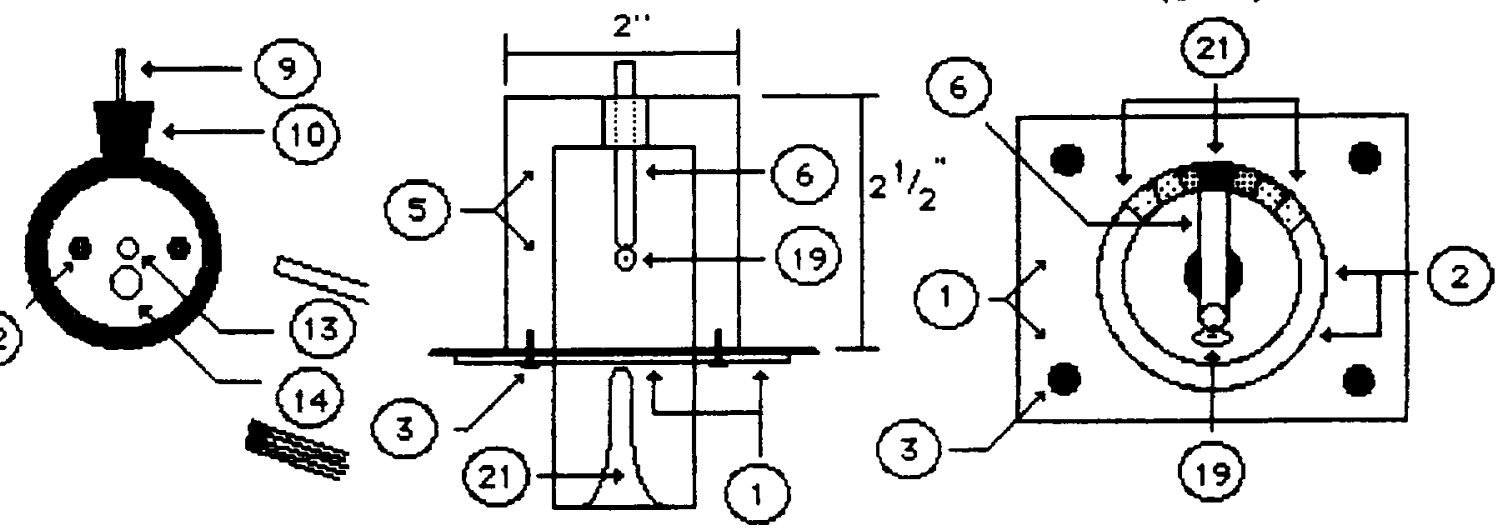

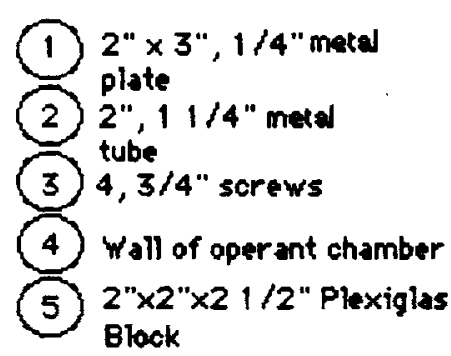

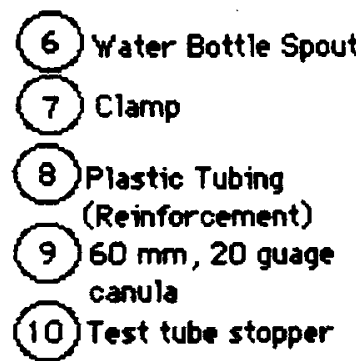

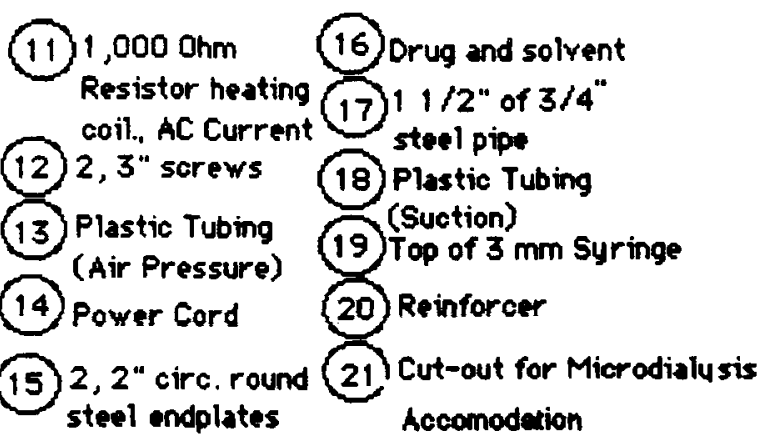

Figure 2. Close-up representation detailing construction of drug volatilization chamber and drug administration chamber. Dimensions of drug administration chamber are for rats weighing between 250 and $500 \mathrm{~g}$.

Once responding has stabilized, the drug may be added. At this point, the animal is responding for food/ water and drug at the same time. Figure 3 shows a typical response pattern obtained at this point in the shaping protocol.

The data in Figure 3 are from a single rat responding on a fixed-ratio (FR10) schedule of reinforcement. Re- sponding is being reinforced with condensed milk and pyrolized cocaine base. The data are from the fifth session in which these conditions were in place. Once responding has stabilized for the food/water and drug reinforcer, the food/water reinforcer should gradually be withdrawn until the animal is responding solely for the drug. This behavior is depicted in Figure 4. 
Figure 4 shows data from 2 rats responding on an FR10 schedule of reinforcement. Responding is being reinforced with pyrolized cocaine base. Data for both rats were taken from the fifth session of responding solely for pyrolized cocaine base.

Roll, McSweeney, See, Kelly, and Meil (1993) have provided additional evidence of the efficacy of this apparatus in a series of studies designed to examine the influence of certain drugs on response patterns.

\section{DISCUSSION}

One problem encountered in any self-administration of respirable pharmaceuticals is the quantification of dose. Individual subjects inhale differently and consequently receive different amounts of the drug. The amount of the drug that is deposited on the nasal membrane instead of entering the respiratory system also varies from subject to subject. This makes the use of within-subjects designs imperative so that each subject may serve as its own control. Although it is not possible to specify dose information a priori, it is possible to quantify drug levels in individual subjects via assaying plasma or urine for either the drug or its metabolites. Another option for quantifying drug levels is to use a microdialysis procedure.

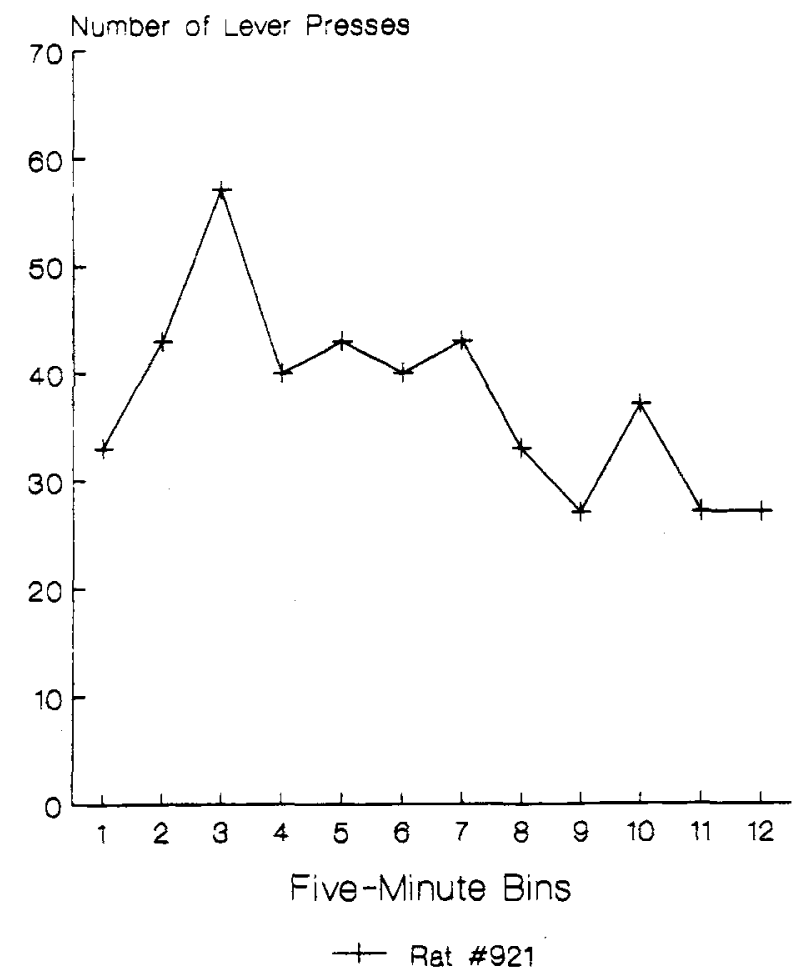

Figure 3. Response rate for a single rat responding during a single 1-h session for both condensed milk and pyrolized cocaine base. Delivery of reinforcers was programmed to occur on a fixed-ratio (FR10) schedule of reinforcement. The session has been broken down into twelve 5-min bins to permit examination of molecular, within-session response patterns.

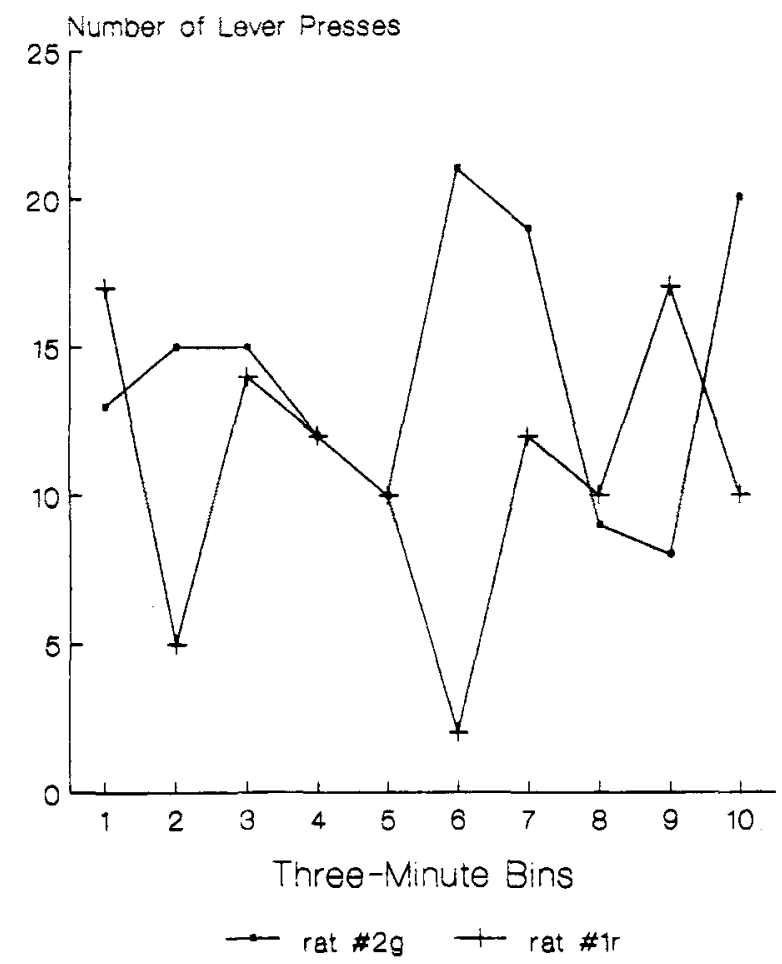

Figure 4. Response rate for 2 rats responding for pyrolized cocaine base. Delivery of reinforcers was programmed to occur on a fixedratio (FR10) schedule of reinforcement. The session has been broken down into ten 3-min bins to facilitate the examination of within-session patterns of responding.

Although the difficulty in quantifying dose does dictate the design of studies that can be conducted with this device, it does not limit the utility of the device. Use of the device described in this paper should facilitate research efforts in basic behavioral pharmacology.

\section{REFERENCES}

Chaiken, M. R. (1993, April). Can drug epidemics be anticipated? National Institute of Justice Journal, pp. 23-30.

BoNi, J. P., BARR, W. H., \& MARTIN, B. R. (1991). Cocaine inhalation in the rat: Pharmacokinetics and cardiovascular response. Journal of Pharmacology \& Experimental Therapeutics, 257, 307-315.

Burchfield, D. J., Abrams, R. M., Miller, R. L., \& DeVane, C. L. (1991). Inhalational administration of cocaine in sheep. Life Sciences, 48, 2129-2136.

JaFFe, A. B., Sharpe, L. G., \& JAFFe, J. H. (1989). Rats selfadminister sufentanil in aerosol form. Psychopharmacology, 99, 289-293.

PrusaczyK, W. K., \& Fischer, G. J. (1983). A small-animal inhalation chamber for short-to-intermediate term exposure. Behavior Research Methods \& Instrumentation, 15, 369-373.

Roll, J. M., McSweeney, F. K., See, R. E., Kelly, B. P., \& Meil, W. (1993). Within-session variation in behavior: An untapped source of data and potential confounds for behavioral pharmacology. Society for Neuroscience Abstracts, 19, 1610.

(Manuscript received July 2, 1993; revision accepted for publication March 22, 1994.) 